\title{
Push-based XML access control policy languages: a review
}

\begin{abstract}
Several access control policy languages have been proposed for specifying access control policies for push-based XML access control systems. This paper investigates the scalability of the current XML-based policy languages. It starts by introducing the well-known general access control models with more focus on their scalability. Then, the XML-based policy languages are presented followed by evaluating their management and system scalability. This paper founds that there is a need for using decentralized trusted management concept for addressing the scalability issue in XML access control. Also, using IBE will help in providing several access control features as temporal and delegable access.
\end{abstract}

Keyword: Access control; Decentralized trust management; Policy specification; Scalability; XML 